\title{
Die Ökonomisierung der Bildung ist eine Herausforderung. Es gilt, sie anzunehmen, mit etwas Gelassenheit.
}

\section{Gianni Ghisla}

\begin{abstract}
"Die betroffenen Händler und Chefs missachteten Grösse und Qualität der Positionen, und die zentralen Risikomanager kannten zwar die Zablen, wussten diese aber nicht richtig zu interpretieren.» Marcel Ospel ${ }^{1}$
\end{abstract}

Immer wenn das Selbstverständliche nicht mehr selbstverständlich ist, schlägt die Stunde der Philosophie.»

Georg Friedrich Hegel

In diesem Beitrag wird für eine kritische Betrachtung von Standards, v.a. von Performance-Standards plädiert, zugleich aber auch eine Lanze für eine notwendige selbstkritische Reflexion gängiger erziehungswissenschaftlicher Praxis gebrochen. Erziehungswissenschaft sollte nicht in die Opferrolle rücksichtsloser Politik hineinmanövrieren, sondern aktiver versuchen, relevante gesellschaftliche Umwälzungen in ihren Blick zu nehmen und handlungsorientiert zu verarbeiten. Dies gilt z.B. für die neu zu konzipierende Beziehung zwischen Bildung, Gesellschaft und Arbeit, wodurch das "Ökonomische " und damit auch die Kategorie der Standards ins Zentrum des Interesses rücken. Es kann also schlicht nicht darum gehen, Standards aus kritischer Warte abzulehnen, weil sie eine ökonomisierende Marklogik mit ihrer instrumentellen Vernunft repräsentieren, denn damit ginge man an der historischen Realität vorbei. Vielmehr gilt es, sie in eine neue Perspektive kompetenzorientierter Pädagogik und Didaktik zu integrieren.

\section{Incipit}

Im Beitrag von Herzog geht es um den Status von Erziehungswissenschaft und Bildungsforschung im aktuellen gesellschaftlichen Kontext, es geht aber auch um die Ziele und die zentralen Kategorien der zur Zeit anbrechenden Bildungsreformen und um das, was Erziehungswissenschaft und Bildungsforschung dazu 
zu sagen haben. Offensichtlich ist diesbezüglich der Begriff «Standard» eine Schlüsselkategorie.

Man kann sich provokativ auf dem Wege zur 08/15-MaschinengewehrSchule wähnen oder die Quintessenz des Standarddiskurses in der Schulbildung plakativ wie folgt auf den Begriff bringen:

«Standardization reduces the quality and quantity of what is taught and learned at school» (McNeil, 2000, S. 3).

Aber macht es Sinn mit solchen Statements zu operieren? Es gibt gute Gründe dafür und dagegen. Dafür spricht, dass man vielleicht die mitunter desillusionierte und träge gewordene Gemeinschaft der Erziehungswissenschaftler und Pädagogen aufrütteln sollte. Dafür spricht auch, dass es unterdessen genügend so genannte Evidenzen gibt, welche die negativen kurzfristigen und langfristigen Auswirkungen von "performance-standards» aufs Lernen, auf das Schulklima und auf die soziale Gerechtigkeit datenbasiert aufzeigen ${ }^{2}$. Dafür spricht schliesslich auch die Deutlichkeit dieser Statements, welche den beinahe magisch-religiösen Erwartungen all jener gegenübergestellt wird, die ihren Glauben an eine Verbesserung des Lernens und an eine bessere Steuerung der Schule mit der Standardisierung gekoppelt haben. Dagegen spricht aber das Risiko der damit verbundenen Ideologisierung des Diskurses und des Verlustes der notwendigen, kritischen Einstellung, die alleine eine rationale, wissenschaftliche Auseinandersetzung ermöglichen kann. Dagegen spricht auch die damit einhergehende Verhärtung der Fronten, welche sich nicht zuletzt auf die wünschbare Gelassenheit des wissenschaftlichen Tuns auswirkt ${ }^{3}$.

Dass damit eine für die Wissenschaft selbstkritische Einstellung gegenüber den sogenannten Evidenzen einher gehen muss, hat Herzog mit allem Recht hervorgehoben. Der Verlust dieser reflexiven Kritik, d.h. der Anwendung des radikalen cartesianischen Zweifels auf sich selbst, zugunsten verfahrensbasierter Selbstsicherheit, ist für den wissenschaftlichen Betrieb nicht gerade verheissungsvoll. Es wäre deshalb Weise und im Interesse aller, v.a. des wissenschaftlichen Betriebs, empirischen Evidenzen ihren Absolutheits- und Selbstverständlichkeitsanspruch zu nehmen, um sie wieder in den Bereich einer kritisch-interpretativen Praxis zurückzuführen.

\section{Herzogs zweifelhafte Architektur des Opfers}

Über die Notwendigkeit Herzogs Polemik als Beitrag zur erwähnten kritischinterpretativen Praxis kann kein Zweifel bestehen. Ceci dit, fallen bei der inneren Architektur seines Textes drei Momente auf. Ein Moment der Anklage («die aktuelle Reform kann der Erziehungswissenschaft und Bildungsforschung Schaden zufügen», "die Amputation der Wissenschaft um ihre theoretische und kritische Dimension bedeutet, ...», usw.), das zugleich die Rolle der Wissenschaft als Opfer rücksichtsloser Politik präfiguriert. Ein Moment der Analyse, welches 
die Begriffe und Kategorien der Reform bzw. der aktuellen nicht nur schweizerischen Bildungspolitik skizziert. Und schliesslich das fehlende Moment der pars costruens, das zwar gar nicht zu einem Pamphlet dieser Art gehören muss, hier aber als Aussichtslosigkeit der Architektur des Opfers daher kommt und sich in einem etwas gar fragilen Hinweis auf die Vorteile des Vertrauens gegenüber der Kontrolle erschöpft.

Die Rolle des Opfers steht der Wissenschaft schlecht an. Das politische System verfügt über genügend Legitimation, um, zur Durchsetzung der eigenen Interessen, die wissenschaftlichen Befunde beanspruchen zu können, und die Wissenschaft müsste ihrerseits selbst dafür sorgen, sich nicht ausnützen zu lassen. Aber dies ist wohl nur die halbe Wahrheit, zumal sich niemand, auch die Wissenschaft nicht, trotz ihrer privilegierten Position, der Wirkung machtpolitischer Verhältnisse entziehen kann. Trotzdem ist die Rolle des Opfers nicht dazu geneigt, die Wissenschaft, insbesondere die Erziehungswissenschaft und die Bildungsforschung, aus einer tatsächlich nicht beneidenswerten Position einer billigen ancilla politicae heraus zu holen.

Es ist kaum von der Hand zu weisen, dass Pädagogen u.a. aufgrund ihres normativen und reproduktiven Geschäfts zur Opferrolle neigen und dadurch in der Öffentlichkeit wie in der Politik nicht unbedingt mehr ernst genommen werden und zu höherem Respekt gelangen. Deshalb wäre es vermutlich für die Erziehungswissenschaft ${ }^{4}$ sinnvoll, eine selbstkritische Attitüde einzunehmen und einmal radikal über die Bücher zu gehen. Was hat sie in den letzten Jahrzehnten getan, um neue oder sich anbahnende Realitäten, die sogar den Charakter epochaler Umwälzungen anzunehmen scheinen, zu erfassen und sich als wegweisende Handlungswissenschaft zu profilieren? Was hat diese Erziehungswissenschaft getan, um sich Gehör zu verschaffen, nicht nur in der Politik, sondern v.a. auch bei den Lehrkräften und in der Öffentlichkeit? Schaut man sich herum, bekommt man zwar allerlei Bemühungen um die Erneuerung des pädagogischen Diskurses zu Gesicht ${ }^{5}$, aber es sind eben andere Elemente, die die Identität und das Image der Erziehungswissenschaft prägen (v.a. bei den Hauptbenutzern, den Lehrkräften $)^{6}$. Man hat zugewartet, bis ökonomisch motivierte Institutionen wie die OECD die Initiative ergriffen haben, und mit ihren sinnvollen Daten einen guten Teil des Feldes besetzt haben. Nach der Reformeuphorie der 70er Jahre wurde die Struktur- und Orientierungsebene verlassen und man hat sich u.a. schnell ins Reduit der Mikroprozesse der Schule zurückgezogen, ohne zu bemerken, dass draussen in rasendem Tempo Umwälzungen statt fanden und die Ökonomisierung rasch Oberwasser erhielt. Es macht deshalb nicht viel Sinn, sich über «die Amputation der Wissenschaft um ihre theoretische und kritische Dimension» zu beklagen, wie dies Herzog tut. Sinnvoller wäre es, sich um u.a. das "Ökonomische» intensiv zu kümmern, das die Welt weitgehend zu beherrschen scheint ${ }^{7}$, und auch um die Bildungsökonomie, die wieder mächtig in den schulischen Garten eindringt. Vielmehr gälte es, die Herausforderung anzunehmen und die neue Beziehung zwischen Arbeitswelt, Bildung und Gesellschaft zu 
einem zentralen Thema zu machen, wie dies einige bereits getan haben (Hoffmann, 1999; Hoffmann \& Maack-Rheinländer, 2001; Oelkers, 2003a, 2003b). Die Kategorien der Schulreform, in primis Standard und Kompetenz, die zum Gerüst dieser neuen Wirklichkeit gehören, bergen in sich eine ökonomische Dimension, um die man nicht herum kommen kann. Sie sind für die Erziehungswissenschaft nicht zuletzt deswegen besonders interessant, da sie einen hohen Gehalt an Ambiguität aufweisen, d.h. sie können je nach Auslegung und Entwicklungstendenz durchaus ein, aus pädagogischer und humanistischer Perspektive, positives wie negatives Potential entfalten.

\section{Apropos ókonomisierung}

Economy is the art of making the most of life. George Bernard Shaw

Die Ökonomisierung beherrscht zusehends die Welt: Sie prägt den Alltag und die Kultur, sie bestimmt Politik, Krieg und Frieden und hat der Logik der instrumentellen und technischen Vernunft (Weber, 1921) zu einem undenkbaren Siegeszug verholfen.

Gary S. Becker, Nobelpreisträger und Mitbegründer der modernen Bildungsökonomie, hat den vermutlich konsequentesten Versuch gemacht, das Diktum von G. B. Shaw ernst zu nehmen und das menschliche Verhalten aus der ökonomischen Perspektive zu verstehen und zu erklären ${ }^{8}$ :

The economic approach is clearly not restricted to material goods and wants, nor even to market sector. (...) Indeed, I have come to the position that the economic approach is a comprehensive one that is applicable to all human behaviour, be it behaviour involving money prices or imputed shadow prices, repeated or infrequent decisions, large or minor decisions, emotional or mechanical ends, rich or poor persons, men or woman, adults or children, brilliant or stupid persons, men or women, businessmen or politicians, teachers or students. (Becker, 1976, S. 6 u. 8) ${ }^{9}$

In brillianter Weise hat der amerikanische Ökonom den Nagel auf den Kopf getroffen und eine willkommene Legitimation für den sich diffundierenden Zeitgeist der Ökonomisierung geliefert. Problematisch ist aber keineswegs die ökonomische Betrachtungsweise an sich, problematisch ist deren Hegemonie. Es geht also nicht um die simple Übernahme von Standards aus der Industrie, wie dies Herzog zu suggerieren scheint. Es geht um die Ökonomisierung von Bildung und Erziehung als Teil eines Gesamtprozesses. Dazu müssen wir wiederum Becker interpellieren, denn, zusammen mit Theodore W. Schulz (auch Nobelpreisträger), gilt er als Begründer der modernen Bildungsökonomie (Becker, 1993 [1964]), jener Disziplin, die speziell Bildung von einem Konsum- zu einem Investitionsobjekt gemacht und den diesbezüglich aussagekräftigen Begriff des Humankapitals geprägt hat. Es kann hier nicht darum gehen, den bildungsökonomischen Diskurs aufzuarbeiten ${ }^{10}$, sondern lediglich festzustellen, dass die- 
ser Diskurs auch in der Schweiz in den letzten zwei Jahrzehnten systematisch Fuss gefasst hat und immer mehr Beachtung findet ${ }^{11}$. Grund genug, so scheint mir, dass sich die Erziehungswissenschaft damit auseinandersetzt und die Aufgabe nicht einfach den Ökonomen überlässt.

\section{Apropos von Standards und Kompetenzen}

Ökonomie hat vorerst mit sparsamem Umgang mit dem Geld zu tun, d.h. das Ökonomische hat primär eine quantitative Seele, eine Seele, die sich ab dem 17. Jahrhundert im Gleichschritt mit dem Höhenflug der messbarkeitsbezogenen Betrachtung der Wirklichkeit entfalten konnte. Die Erfolge positivistischer Wissenschaft haben diesem Paradigma zu ständiger Anerkennung verholfen, in Technik, Wirtschaft, Medizin, usw. Es ist dieses Paradigma, das den heutigen Zeitgeist weitgehend dominiert. Die Kategorie der Standardisierung ist eine der wichtigsten Pfeiler dieser Weltbetrachtung und Weltgestaltung. Standards begleiten uns auf Schritt und Tritt im Alltag, sie erleichtern nicht nur unser Leben, sie ermöglichen es geradezu, indem sie u.a. für die Zuverlässigkeit all dessen sorgen, was wir zum Leben brauchen.

Standard steht für Beispiel, Modell, Massstab, ursprünglich vom Englischen Fahne, Standarte, also etwas, das deutlich ist, symbolische Kraft ausstrahlt und sichtbar und fest steht. Ein Standard repräsentiert eine bestimmte Qualität, die sich punktuell auf einem Qualitätskontinuum festsetzen lässt, weil damit etwa die geforderte Zuverlässigkeit einer Ware oder einer Leistung gewährleistet werden kann. Ein Standard zeichnet sich so mit seiner unverrückbaren Verbindlichkeit aus, es ist Modell, Vorbild und Norm zugleich. ${ }^{12}$

Was bezweckt nun die Standardisierung im Bildungsbereich?

Durch die Standardisierung soll explizit, präzisiert und operationalisiert werden, welche Kompetenzen Lernende (...) zu (einheitlich) festgelegten Zeitpunkten ihrer Lernbiographie auf bestimmten Fachgebieten (in der Regel) entwickelt haben sollen. Standards operationalisieren nicht nur das unterrichtspraktisch zu Erstrebende in der Form erwünschten Lernoutputs, sie bezwecken auch die Vereinheitlichung wünschenswerten Lernoutputs. (Heid, 2007, S. 32)

An Deutlichkeit lässt diese Formulierung nichts zu wünschen übrig: In der Vorstellung all jener, die sie als die Lösung der Qualitätsprobleme der Schule betrachten, sind Standards im Bildungsbereich mit Normierung, Vereinheitlichung, Homogenisierung des Lernens gleichzusetzen. So aufgefasst sind Standards nichts Anderes als die Fortentwicklung der operationalisierten Lernziele alter Schule, auch weil deren Formulierung als Can Do Statement vorgenommen werden muss, also die Verwendung von Verben verlangt, die beobachtbare Handlungen wiedergeben (Oelkers, 2005). Also nichts anderes als alter Wein in neuen Schläuchen? Nein, denn um bei der Metapher zu bleiben, der Wein erhält doch eine neue Qualität, er wird zu korrosivem Essig, und zwar da- 
durch, dass die Verbindlichkeit Ausschliesslichkeitscharakter hat, denn Standards müssen um jeden Preis erreicht werden, «während Ziele bereits ihrem Begriff nach einräumen, dass sie verfehlt (...)» (Ruhloff, 2007, S. 55) oder zumindest nur annähernd erreicht werden können. Es ist auch kein Zufall, dass man von so genannten «high-stake» Standards spricht, die radikale, in der Regel existenzgefährdende, finanzielle Sanktionen nach sich ziehen können. Prinzipiell können als Standard und die Standardisierung des Lernoutputs den Druck auf die Bildungsprozesse soweit verstärken, dass sie die Existenz einer Bildungsinstitution oder einer beruflichen Karriere in Frage stellen. Müssen sie deshalb von den Pädagogen auch prinzipiell abgelehnt werden? Die Frage hat ihre Berechtigung und Herzog scheut es nicht, zu deren Beantwortung die Register einer gewissen Rhetorik zu ziehen, wenn er einerseits die Empörung auslösende Annäherung von Bildung und «M.G. 08/15» suggeriert und andererseits den in vielen pädagogischen Kreisen verfemten Behaviourismus bemüht.

Es gilt für einen Standard, dass er primär ein Ausdruck von Quantität bzw. einer festen Gleichheitsnorm ist, zugleich aber auf eine bestimmte Qualität bezogen ist, die sich auf einem Qualitätskontinuum festhalten lässt. Dies gilt jedoch nicht umgekehrt, d.h. dass nicht jede Qualität (oder jedes qualitative Phänomen) auf eine Quantität Bezug nehmen und standardisierbar sein muss (Ruhloff, 2007, S. 51), denn es gibt Qualitäten, die den Status der Einmaligkeit und der Unnachahmbarkeit haben. Bildung und pädagogisches Handeln sind menschbezogene Prozesse, die an sich diese Einmaligkeit widerspiegeln und, wie von Herzog zu Recht bemerkt, nicht technisierbar sind. Dies gilt sowohl für die Lehr- und Lernprozesse als auch für deren Ergebnisse, nämlich das, was sich Lernende angeeignet haben.

Die Diskussion, so scheint mir, kann sich nur dann nicht verfahren, wenn man sich den Sinn und die Zielsetzung der Standardanwendung vergegenwärtigt und unterscheidet zwischen i) der Art der Standards und ii) des Objekts bzw. der Ebene, auf welcher Standards und Standardmessungen angewendet werden ${ }^{13}$. Man kann einen breiten Konsens darüber voraussetzen, dass die Qualität der Schule verbessert werden kann und muss und dass dabei die Schule und deren Akteure zu einer angemessenen Rechenschaft herangezogen werden müssen. Wenn dies die Zielsetzung ist, dann sollte man die Art der Standards unterscheiden und ziel- bzw. objektadäquat einsetzen. Es kann nicht darum gehen, nur auf out-put orientierte "performance standards» zu setzen, sondern auch «opportunity-to-learn standards», «content standards» usw. sollten zur Anwendung kommen. Insbesondere aber können "performance-standards» nicht auf die Mikroebene des Bildungsprozesses angewendet werden, denn dort treffen sie genau auf die Einmaligkeit und nicht Technisierbarkeit von Bildungsprozessen. Wie es auch Klieme deutlich und klar vorgegeben hat (Klieme et al., 2003) "performance standards» dürfen nicht zur Beurteilung der Leistung von einzelnen Lernenden angewendet werden, sie dienen dem Monitoring des Macrosystems, allenfalls des Mesosystems und können als solche, mit anderen Indikatoren, zu 
einer fruchtbaren Auseinandersetzung, zur Systemerneuerung beitragen und damit auch ein positives Potential entwickeln. Natürlich kann man diesbezüglich leicht der Naivität bezichtigt werden ${ }^{14}$. PISA docet, dass der Zeitgeist leicht Überhand gewinnt, aber es ist auch so, dass die grossen Probleme der Standardisierung mit der Umsetzung ins Testing und deren gewinnorientierten Nutzung durch private Anbieter, die den Weg zum «teaching and learning to the test» bereitet haben. Für eine differenzierte Anwendung der Standards hat sich auch die EDK festgelegt, aber, wie Herzog vermerkt, sie müsste auch dafür sorgen, dass man davon nicht abweicht. Trotzdem, bleibt m.E. den Erziehungswissenschaftlern und den Pädagogen, die ihre Autonomie wahren und sich nicht zu verlängerten Armen von Ökonomie und Politik degradieren lassen wollen, nichts anderes übrig, als sich dieser Herausforderung zu stellen und u.a. für eine sinnvolle, differenzierte und den Lernprozessen adäquate Anwendung von Standards einzustehen.

Damit kann kurz auch der Kompetenzbegriff zur Sprache gebracht werden. Mit den (Performance-) Standards sollen Kompetenzen identifiziert und so operationalisiert werden, dass sie messbar werden. D.h. also, dass «nicht etwa schulisches Wissen», sondern eben Kompetenzen gemessen werden sollen, die für bestimmte Fachbereiche ${ }^{15}$ schweizweit bestimmt werden. Herzog meidet die entscheidende Frage der Kompetenzen, m.E. zu Unrecht. Geht man diese im Überblick an, so stellt man Interessantes fest, und ich konzentriere mich dabei auf die Diskussion im deutschsprachigen Sprachraum, wo seit den 70er Jahren ein Kompetenzbegriff die Vorherrschaft hat, der gerade von den Erziehungswissenschaftlern einfach unkritisch und ohne jegliche ernsthafte Bemühung um theoretische Aufarbeitung hingenommen wurde ${ }^{16}$ : Dieser Begriff nimmt auf einen anthropologischen, subjektorientierten Begründungszusammenhang Bezug und unterscheidet zwischen Fach-, Methoden und Sozial- bzw. Personalkompetenzen. Gleichzeitig wird gesagt, dass Kompetenzen eben Dispositionen seien, die die Bewältigung von konkreten Anforderungssituationen ermöglichen (z.B. Klieme et al., 2003). Dabei wird seltsamerweise übersehen, dass man mit Fachkompetenzen alleine, genau gleich wie mit Methoden- oder Sozialkompetenzen alleine, gar keine konkrete Anforderungssituation bewältigen kann, denn dazu braucht es ein Konglomerat all dieser Kompetenzen. Bei näherer Betrachtung kann also eine Fachkompetenz gar keine Kompetenz sein, denn mit fachlichem Können allein ist keine Situation zu meistern. Es braucht dazu immer auch andere Ressourcen, also auch «Methoden- und Selbstkompetenzen». Die Verwechslung der zwei logischen Ebenen ist frappant. Mit einer rhetorischen Figur ausgedrückt, liegt eine Synekdoche vor: pars pro toto (Boldrini \& Ghisla, 2006) ${ }^{17}$. Sinnvoller scheint mir hingegen, diese Unterscheidung nicht nur zu beachten, sondern zu einer konstitutiven Begriffskomponente zu machen, denn damit kann mit einem sauberen und theoretisch konsistenten Kompetenzbegriff operiert werden, der zwischen situationsorientierten Kompetenzen und subjekt- 
orientierten Ressourcen (d.h. Dispositionen) unterscheidet. Als Kompetenz ${ }^{18}$ wird demnach

zuerst einmal die Fähigkeit von individuellen oder kollektiven Subjekten verstanden, eine Klasse von Situationen, allenfalls eine einzelne Situation, erfolgreich zu meistern und somit eine Tätigkeit zu vollziehen. Um in Situationen kompetent zu sein, bedarf es der Aktivierung von individuellen oder gruppengebundenen Ressourcen, nämlich von Kenntnissen, Fähigkeiten und Haltungen.

Mit dieser Definition wird von einem rein subjektorientierten Kompetenzbegriff Abstand genommen, d.h. Kompetenzen gelten als situationsbezogen, sie sind einmalig, entstehen in Situationen und sind nur situations- und kontextbezogen beschreibbar, während Ressourcen subjektspezifisch sind und dem Potential entsprechen, das dem Subjekt innewohnt. Die Konsequenzen dieser kritischen Fassung des Kompetenzbegriffs ${ }^{19}$ sind noch kaum überblickbar, aber schon daran ablesbar, dass die allgemeinbildende, obligatorische Schule zwar kompetenzorientiert arbeitet, aber nur in sehr geringem Ausmass Kompetenzen vermitteln kann und sich wesentlich auf die Ressourcen, d.h. auf Kenntnisse, Fähigkeiten und Haltungen konzentrieren sollte ${ }^{20}$. Dies bedeutet auch, dass es kaum Sinn macht, den Output der Schule ausschliesslich in Form von Kompetenzen beurteilen zu wollen.

Die Entwicklung von curricularen Konzepten, die von diesem Kompetenzbegriff ausgehen ist durchaus möglich, bereits erprobt und implementiert wor$\operatorname{den}^{21}$. Solche Konzepte zeigen auf, welches Potential im Kompetenzbegriff schlummert, sofern man ihn nicht reduktiv, entweder im Sinne des umgangssprachlich im Deutschen verwendeten Terminus oder im Sinne des englischen «skill» verwendet ${ }^{22}$ (Ghisla, 2007).

\section{Wider die Instrumentalisierung der Erziehungs- wissenschaft - ja, aber...}

Es besteht kein Zweifel darüber, dass die ökonomisierende, die instrumentelle Vernunft beanspruchende Seele des Zeitgeistes, die Bildungspolitik dazu «verführt» hat, die notwendigen Reformen und Verbesserungen des Bildungssystems de facto mit Mitteln und Strategien anzustreben, die wesentlich wettbewerbsorientiert sind und auf Messbarkeit und Vergleichbarkeit der erzielten Resultate abstellen. Mann muss sich vergegenwärtigen, dass dieser Zugang an sich keineswegs neu ist, sondern von der in der Nachkriegszeit eingesetzten Curriculumdiskussion mit der Festlegung von Lernzielen und den daran anschliessenden Kontrollverfahren bereits prinzipiell und in concreto angelegt worden war. Die heutige Entwicklung signalisiert eine Radikalisierung. Von Kontrollmechanismen, die sich auf die Marktlogik, auf nationale und internationale Konkurrenzfähigkeit sowie auf Messbarkeit abstützen, erwartet man effizientere Steuerungsmöglichkeiten des Systems. Über den Mechanismus der Rechenschaftsablegung 
und der Ranglistenvergleiche, sollen die wichtigen Akteure, auf allen Ebenen des Systems, d.h. von der Lehrkraft zum Bildungsminister, derart unter Druck gesetzt werden, dass Optimierungseffekte im ökonomischen Sinne erwartet werden. Es lohnt sich, diesbezüglich einen Blick auf die Richtlinien des Bundesrates (Bundesrat, 2007) und auf die «Botschaft über die Förderung von Bildung, Forschung und Innovation in den Jahren 2008-2011» zu werfen (Consiglio federale, 2007). Die Richtlinien selbst sprechen unmissverständlich bereits im Titel von "Erhöhung der Wettbewerbsfähigkeit und des Wachstums», worauf die Sicherung und Steigerung der Qualität des Bildungswesen auszurichten sei. Damit ist die ökonomische Finalisierung von Bildung, Forschung und Innovation unmissverständlich festgelegt und die Bildungspolitik hat sich danach auszurichten ${ }^{23}$. Wenn es dann um die Prioritäten und die Massnahmen geht, ist aber die Marschrichtung klar: Effizienzsteigerung und Wettbewerbsfähigkeit stehen im Vordergrund, wobei «der Wettbewerb um die Mittel (...) die Selbststeuerung des gesamten Bildungs-, Forschungs- und Innovationssystems (...) beschleunigen wird» (ibid, 1229). Solche Eckwerte der Bildungspolitik müssen sich Erziehungswissenschaft und Bildungsforschung vor Augen führen, daran können und sollen sie nicht vorbeigehen. Mit den damit einhergehenden Prozessen müssen sie sich m.E. systematisch befassen. Dazu gehören u.a. zweierlei Phänomene, die Herzog indirekt thematisiert: Eine Art «Kulturbereinigung», die Inhalte und Bildung im traditionellen, humanistischen Sinne in den Hintergrund drängt, und die «Neutralisierung der Einmaligkeit», welche jedem Bildungsprozess und -ergebnis eigen ist ${ }^{24}$. Ein guter Teil der Erziehungswissenschaft und Bildungsforschung verschliesst sich diesen Prozessen, oder aber macht und gestaltet den Trend mit Überzeugung (oder bereitwillig) mit.

Diese Probleme haben an sich sehr wenig mit dem berechtigten Bestreben zu tun, auf der Basis der nun verfassungsmässig abgestützten Kompetenzen des Bundes, einen schweizerischen Bildungsraum zu schaffen und eine notwendige Koordination und Harmonisierung einzuführen, die die kantonale oder regionale Autonomie keineswegs beschneiden muss. Vielmehr ginge es um die Fähigkeit der Bildungspolitik, Bildung nicht zur Ware degradieren zu lassen und das Bildungssystem vor dem Abgleiten in ein betriebliches, ausschliesslich den Zwängen des Wettbewerbs ausgelieferten Produktionssystems zu bewahren. Leider verfügen Erziehungswissenschaft und Bildungsforschung, aber auch generell die Sozialwissenschaft in diesem Konzert über keine «erste Geige». Dezidierte Appelle an die Macht (lies: Bildungspolitik) wider die Instrumentalisierung sind zwar durchaus legitim, vielleicht notwendig, aber realistischerweise bringen sie nicht viel. Erziehungswissenschaft und Bildungsforschung müssen aus ihrer Lethargie herausfinden und sich mit neuen Realitäten konfrontieren, insbesondere die Bildung im Verhältnis zwischen Arbeit, Kultur und Gesellschaft überdenken und dazu neue Ansätze entwickeln, die die Ökonomisierung und Technisierung kritisch aufnehmen. Aber bitte: auch mit einer Prise Gelassenheit! 


\section{Anmerkungen}

1 Chef der Grossbank USB, anfangs Dezember 2007 vor Investoren in London, jegliche Verantwortung für die Subprime-Krise von sich weisend. Unterdessen hat die UBS bereits 21 Milliarden abgeschrieben.

2 Auf die entsprechende Literatur verweist auch Herzog.

3 Dies soll nicht bedeuten, dass Wissenschaft grundsätzlich von Verantwortungs- und Parteinahme entbunden sei (Jonas, 1984).

4 Ich bin mir bewusst, dass der Terminus Erziehungswissenschaft expliziert werden müsste. Da dies hier nicht möglich ist, seien immerhin Pädagogik, Didaktik und die Sozialwissenschaften, soweit sie sich mit dem Gegenstand Bildung und Erziehung beschäftigen, dazu gezählt.

5 Vgl. für die Schweiz einige der von der Schw. Gesellschaft für Bildungsforschung durchgeführten Kongresse oder andere Symposien, die zu bedeutsamen Publikationen geführt haben (Hansen, Sigrist, Goorhuis \& Landolt, 1999; Oelkers, 2003b). Man vergesse aber auch nicht, dass eines der wichtigsten Forschungsprogramme der letzten Jahre, das Programm 33 zur Wirksamkeit der Bildung, sich eben auf Fragen der Effizienz und Effektivität konzentriert hat.

6 Etwas salopp ausgedrückt: Konstruktivistische Selbstbewunderung, reformpädagogische und rezeptorientierte Rigidität, Verteidigung à tout prix eines problematischen Bildungsbegriffs, Duckmäusertum gegenüber der Empirie, usw. haben Pädagogik und Didaktik dazu gezwungen, der Realität ständig nachzurennen, und zwar mehr als es in der Natur der Sache sein dürfte. Man frage bei den Lehrkräften nach, was sie von Erziehungswissenschaft halten. Die meisten, die ich kenne, zeigen z.B. beim Hören des Begriffs «Konstruktivismus», einer der meistverwendeten Termini in der Lehrkräfteausbildung, Symptome mittlerer Bauchkrämpfe und leichterer Brechreize.

7 Vgl. dazu auch die Diskussion in der Öffentlichkeit, z.B. in der NZZ vom 12./13. Januar 2008, mit dem Beitrag «Das 'Ökonomische' beherrscht die Welt».

8 Damit soll der Anspruch der Klassiker der Ökonomie, der Utilitaristen wie Jeremy Bentham, und insbesondere von K. Marx keineswegs geschmälert werden. Hier geht es wesentlich um die neueren Ansätze der Sozialwissenschaft.

9 Um es noch deutlicher zu illustrieren: «According to the economic approach, a person decides to marry when the utility expected from marriage exceeds the expected from remaining single or from additional search for a more suitable mate (...). Similarly, a married person terminates his (or her) marriage when the utility anticipated from becoming single or marrying someone else exceeds the loss in utility from separation, including losses due to physical separation from one's children, division of join assets, legal fees, and so forth.» (Becker, 1976, S. 10) Becker gebärdet sich allerdings vorsichtig: «Although I am arguing that the economic approach provides a useful framework for understanding all human behavior. I am not trying to downgrade the contributions of other social scientists, nor even to suggest that the economist's are more important.» (ibid)

10 Vgl. dazu etwa: Frick \& Wirz, 2006; Grin, 2005, S. 61-150; Wolter, 2002.

11 Der Trend wird durch die Intensivierung der akademischen Aktivität mit Instituten an den Universitäten Basel, Zürich (ETH und Universität), Bern und Genf sowie im neu konstituierten Eidgenössischen Institut für Berufsbildung (EHB in Lausanne, Lugano und BernZollikofen) dokumentiert. Das Bundesamt für Berufsbildung und Technologie (BBT) hat ein sogenanntes Leading House Bildungsökonomie mit der Beteiligung der Universitäten Zürich, Bern und Genf geschaffen. Der Bundesrat hat 2000 das Forschungsprogramm «Bildung und Beschäftigung» lanciert, das zu etlichen Publikationen Anlass gegeben hat (www.nfp43.unibe.ch).

12 Heid sagt es mit aller Deutlichkeit: «Zusammenfassend lässt sich sagen, dass Bildungsstandards i) die Normierung, ii) die Vereinheitlichung, iii) die Überprüfbarkeit und iv) die 
Vergleichbarkeit dessen bezwecken, was Lernende nach dem Durchlaufen bestimmter Bildungsgänge wissen und können sollen». (Heid, 2007, S. 33)

13 Auf die ebenso zentrale Problematik der Messbarkeit von Standards und der damit verbundenen Implikationen wird nicht eingegangen.

14 Handkehrum ist es genauso naiv zu meinen, man könne gegen die Standards einen unter den gegebenen Umständen im Voraus verlorenen, frontalen Kampf führen.

15 Für Harmos sind dies die Unterrichtssprache, die Fremdsprachen, Mathematik und Naturwissenschaften. PISA hat hierfür umfangreiches Anschauungsmaterial geboten.

16 Natürlich sind auch Ausnahmen auszumachen und deswegen soll man Pauschalisierungen vermeiden, aber der Gesamteindruck ist nicht von der Hand zu weisen. Übrigens, die grossen, unterdessen bekannten Probleme, welche bei HarmoS bei der Entwicklung von Kompetenzemodellen für die vier ausgewählten Schulfächer auftauchen, haben nicht zuletzt mit der bisherigen unreflektierten, weitgehend theorielosen Übernahme des geläufigen Kompetenzbegriffs zu tun. Herzog weiss bestens, dass dies nicht das Problem der Politiker ist.

17 Diese logische Verwechslung hat z.B. bei der Übersetzung vom Deutschen in andere Sprachen sonderbare Blüten getrieben: So wurden die Fachkompetenzen ins Italienische und ins Französische mit «competenze professionali «bzw. "compétences professionnelles " wiedergegeben, als Berufskompetenzen, was die Synekdoche noch deutlicher erscheinen lässt.

18 Für eine eingehende Analyse des Begriffs vgl. Boldrini \& Ghisla, (2006).

19 In diese Richtung argumentierte auch Weinert in einer seiner letzten Auseinandersetzungen mit dem Kompetenzbegriff und bewegen sich unterdessen viele andere Autoren auf europäischer Ebene (LeBoterf, 1994, 2000; Rychen \& Salganik, 2003; Rychen, Salganik \& McLaughlin, 2003; Weinert, 2001; Winterton, Deist \& Stringfellow, 2005).

20 Anders liegt die Diskussion bei der Berufsbildung, wo die Ausbildung als Partnerschaft zwischen Schule und Betrieb gestaltet wird.

21 Vgl. dazu: Ghisla, 2002, 2007; Ghisla et al., 2008; Roegiers, 2000.

22 Der im Englischen am meisten verwendete Begriff ist wohl skill, obzwar auch competence und competency durchaus gebraucht werden. Skill bezieht sich spezifisch auf ausführende Fähigkeiten zur technischen Bewältigung von bestimmten Problemen.

23 Auffallend ist immerhin, dass die eigentlichen Zielsetzungen dieser Bildungspolitik ein Bildungssystem anstreben, das «...den sich ändernden Bedürfnissen der Bildungswilligen jeden Alters und den kulturellen, sozialen und wirtschaftlichen Interessen der Gesellschaft» gerecht werden und weiter die «Integration einer möglichst grossen Anzahl von Jugendlichen in das nachobligatorische Bildungssystem» ermöglicht und "die Chance der Integration von schulisch und sozial Schwächeren verbessert» werden soll (Consiglio federale, 2007, S. 1227).

24 Es sei hier kurz eine Anekdote erlaubt. Ich habe kürzlich an einer Pressekonferenz in einem grossen Schweizer Kanton teilgenommen, wo Massnahmen und Strategien zur Eindämmung der Jugendarbeitslosigkeit nach der obligatorischen Schule vorgestellt wurden. Die Organisation war optimal. Neben dem kantonalen Bildungsminister und den hohen Beamten waren Vertreter der Arbeitswelt und der Wissenschaft zugegen, sodass von einer effektiven Partnerschaft der wichtigsten stakeholder gesprochen werden konnte. Studenten konnten zu didaktischen Zwecken auch teilnehmen. Zahlen wurden geliefert, die eine koordinierte und kohärente Strategie zwischen Administration, Arbeitswelt und Wissenschaft stützten und auch bemerkenswerte Resultate dokumentierten. Interessant war auch, dass der zuständige hohe Beamte die Verantwortung für die gesamte Sekundarstufe II hat, sodass eine koordinierte Politik für die Allgemein- und die Berufsbildung möglich wird. Gerade dieser Beamte, der seine Aufgabe managementmässig offensichtlich bestens bewältigt, erlaubte mit seinen klaren und prägnanten Aussagen die hier diskutierte Problematik auf den Begriff zu bringen. Er sagte, dass er für die Politik der Sekundarstufe II 
drei präzise Zielsetzungen habe, die auch allen MitarbeiterInnen so vertraut seien: 1 . Erhöhung der Abschlüsse der Sekundarstufe II auf mehr als 95\% und dies obzwar der Kanton schweizerisch schon an der Spitze liege. 2. Verbesserung der Resultate der Maturitätsprüfungen, sodass man besser als die anderen Kantone da stehen könne. 3. Optimierung der Übergänge von der obligatorischen Schule zur nachfolgenden Ausbildungsstufe.

An sich erübrigt sich ein Kommentar... Die Dominanz quantitativer Zielsetzungen und der Vergleich mit anderen Kantonen als quasi ausschliesslicher Massstab für den Erfolg sprechen eine deutliche Sprache. Es frappiert, dass gerade auf der Sekundarstufe II, einer für die Allgemeinbildung wohl entscheidenden Stufe, die Kultur oder inhaltliche Zielsetzungen irgendwelcher Art augenscheinlich keine zentrale Rolle mehr spielen. Eine offensichtliche «Kulturbereinigung» hat stattgefunden.

\section{Bibliographie}

Becker, G. S. (1976). The Economic Approach to Human Behavior (pp. 6-10) Chicago: The University of Chicago Press.

Becker, G. S. (1993 [1964]). Human Capital. Chicago: The University of Chicago Press.

Boldrini, E. \& Ghisla, G. (2006). Competenza, Compétence, Competence, Kompetenz. Alcuni spunti sul concetto di competenza a partire da un approccio linguistico. In E. Poglia (Ed.), Competenze e loro valutazione in ambito formativo (Vol. Quaderni dell'Istituto). Lugano: Università della Svizzera Italiana (USI).

Bundesrat (2007). Bildung, Forschung und Innovation. Nachhaltige Sicherung und Steigerung der Qualität. Erhöhung der Wettbewerbsfähigkeit und des Wachstums. Bern: Schweizerische Eidgenossenschaft.

Consiglio federale (2007). Messaggio concernente il promovimento dell'educazione, della ricerca e dellinnovazione negli anni 2008-2011 (pp. 1227).

Online 12.2.08 http://www.sbf.admin.ch/bfi2008/index_it.html

Frick, A. \& Wirz, A. (Hrsg.). (2006). Berufsbildungsökonomie: Stand und offene Fragen. Bern: h.e.p.

Ghisla, G. (2002). An Educational Plan to Face the Complexity of Pedagogical Innovation and its strategic Requirements. In M. Rosenmund, A.-V. Fries \& W. Heller (Eds.), Comparing Curriculum-Making Processes (pp. 133-152). Bern: Peter Lang.

Ghisla, G. (2007). Überlegungen zu einem theoretischen Rahmen für die Entwicklung von kompetenzorientierten Curricula.Unveröffentliches Manuskript, Universität Lugano.

Ghisla, G., Bausch, L. \& Boldrini, E. (2008). CoRe - Kompetenzen-Ressourcen: Ein Modell der Curriculumentwicklung für die Berufsbildung. Zeitschrift für Berufs- und Wirtschaftspädagogik (Manuskript unterbreitet).

Grin, F. (2005). Grundzüge der volkswirtschaftlichen Bildungsökonomie. In V. Bank (Hrsg.), Vom Wert der Bildung (S. 61-150). Bern, Stuttgart, Wien: Haupt.

Hansen, H., Sigrist, B., Goorhuis, H. \& Landolt, H. (Hrsg.). (1999). Bildung und Arbeit Das Ende einer Differenz? Aarau: Sauerländer.

Heid, H. (2007). Was vermag die Standardisierung wünschenswerter Lernoutputs zur Qualitätsverbesserung des Bildungswesens beizutragen? In D. Benner (Hrsg.), Bildungsstandards (S. 29-48). Paderborn: Schöningh.

Hoffmann, D. (Hrsg.). (1999). Rekonstruktion und Revision des Bildungsbegriffs. Vorschläge zu seiner Modernisierung. Weinheim: Deutscher Studienverlag.

Hoffmann, D. \& Maack-Rheinländer, K. (Hrsg.). (2001). Ökonomisierung der Bildung. Die Pädagogik unter den Zwängen des 'Marktes'. Weinheim: Deutscher Studienverlag.

Jonas, H. (1984). Das Prinzip Verantwortung. Frankfurt am Main: Suhrkamp.

Klieme, E., Avenarius, H., Blum, W., Döbrich, P., Prenzel, M., Reiss, K. et al. (2003). Zur Entwicklung nationaler Bildungsstandards. Bonn: Bundesministerium für Bildungsforschung (BMBF). 
LeBoterf, G. (1994). De la compétence: Essays sur un attracteur étrange. Paris: Editions d' Organisation.

LeBoterf, G. (2000). Compétence et navigation professionelle. Paris: Editions d'Organisation.

McNeil, L. M. (2000). Contradictions of School Reform. Educational Costs of Standardized Testing (p. 3). New York: Routledge.

Oelkers, J. (2003a). Introduction: The Future of Vocational Education. In J. Oelkers (Ed.), Futures of Education II. Bern: Peter Lang.

Oelkers, J. (2005). Von Zielen zu Standards. Friedrich Jahresheft XXIII, 18-21.

Oelkers, J. (Ed.). (2003b). Futures of Education II. Essays from an Interdisciplinary Symposium. Bern: Lang.

Roegiers, X. (2000). Une pédagogie de l'intégration. Compétences et intégration des acquis dans l'enseignement. Bruxelles: De Boeck \& Larcier.

Ruhloff, J. (2007). Grenzen von Standardisierung im pädagogischen Kontext. In D. Benner (Hrsg.), Bildungsstandards (S. 49-60). Paderborn: Schöningh.

Rychen, D. S. \& Salganik, L. H. (Eds.). (2003). Key Competencies for a Succesful Life and a Well-Functioning Society. Cambridge,Toronto, Bern, Göttingen: Hogrefe \& Huber.

Rychen, D. S., Salganik, L. H. \& McLaughlin, M. E. (Eds.). (2003). Contributions to the Second DeSeCo Symposium. Geneva, Switzerland, 11-13 February, 2002. Definition and selection of key competencies. Neuchâtel: Swiss Federal Statistical Office.

Weber, M. (1921). Wirtschaft und Gesellschaft (Hauptwerke der grossen Denker.). Paderborn: Voltmedia.

Weinert, F. E. (2001). Concept of Competence: A Conceptual Clarification. In D. S. Rychen \& L. H. Salganik (Eds.), Defining and Selecting Key Competencies. Seattle, Toronto, Bern, Göttingen: Hogrefe \& Huber.

Winterton, J., Deist, F. D.-L. \& Stringfellow, E. (2005). Typology of knowledge, skills and competencies: clarification of the concept and prototype. Thessaloniki: Cedefop.

Wolter, S. C. (2002). Bildungsökonomie - Eine Standortbestimmung. Schweizerische Zeitschrift für Bildungswissenschaften, 1, 149-170.

Schlagworte: Ökonomisierung der Bildung, Bildungsökonomie, Kompetenzen, Standard 